\title{
Wasserkraft und Nachhaltigkeit
}

\section{Untersuchungen zur Auswirkung von Stauanlagen (an der bayerischen Donau) auf die Weichholzaue und Entwicklung von Maßnahmen zu ihrer Förderung}

Christina Fehrmann

\section{Zusammenfassung}

Der Beitrag zu einem aktuellen gleichnamigen Forschungsprojekt diskutiert einen Konflikt innerhalb der Nachhaltigkeitssäule „Ökologie“. So stehen Wasserkraftwerke im Spannungsfeld zwischen der Nutzung des fließenden Wassers als CO2-neutrale, regenerative und grundlastfähige Energiequelle einerseits und den negativen Auswirkungen auf den Lebensraum aus dem Fluss und seinem natürlichen Überschwemmungsgebiet, der Aue, andererseits. Speziell betrachtet werden die Auswirkungen auf den stark bedrohten Lebensraumtyp der Weichholzaue. Nach einer Zusammenfassung der bereits bekannten Einflussfaktoren werden die im Projekt herausgearbeiteten Forschungsansätze zur Förderung der Keimungs- und Etablierungsbedingungen von Silberweidenbeständen vorgestellt. Dendrochronologische Untersuchungen in Kombination mit Analysen von Abflussdaten sollen in der Entwicklung einer Recruitment Box nach dem Vorbild von Forschungen aus dem Nordwesten Amerikas münden. Ergänzt wird dieser Ansatz durch die Evaluierung des Einflusses des Neophyten Impatiens glandulifera auf die Verjüngung von Silberweiden.

(C) Der/die Autor(en) 2017

K.-D. Altmeppen et al. (Hrsg.), Nachhaltigkeit in Umwelt,

Wirtschaft und Gesellschaft, DOI 10.1007/978-3-658-14439-5_2 


\section{$1 \quad$ Aktuelle Situation}

\subsection{Einsortierung der Wasserkraft in aktuelle Nachhaltigkeitsmodelle}

Laut gängigen Nachhaltigkeitstheorien wird Nachhaltigkeit erreicht, wenn die drei Dimensionen Ökonomie, Ökologie und Soziales miteinander in Einklang gebracht werden können. Unabhängig davon, ob das "Nachhaltigkeitsdreieck“, das „magische Dreieck“, das allgemein geläufige „Drei-Säulen-Modell“ oder das "gewichtete Säulenmodell“ zur Veranschaulichung genutzt werden, wird dabei suggeriert, dass die jeweiligen Dimensionen in sich einheitliche und konsistente Gebilde sind (s. Abbildung 1).

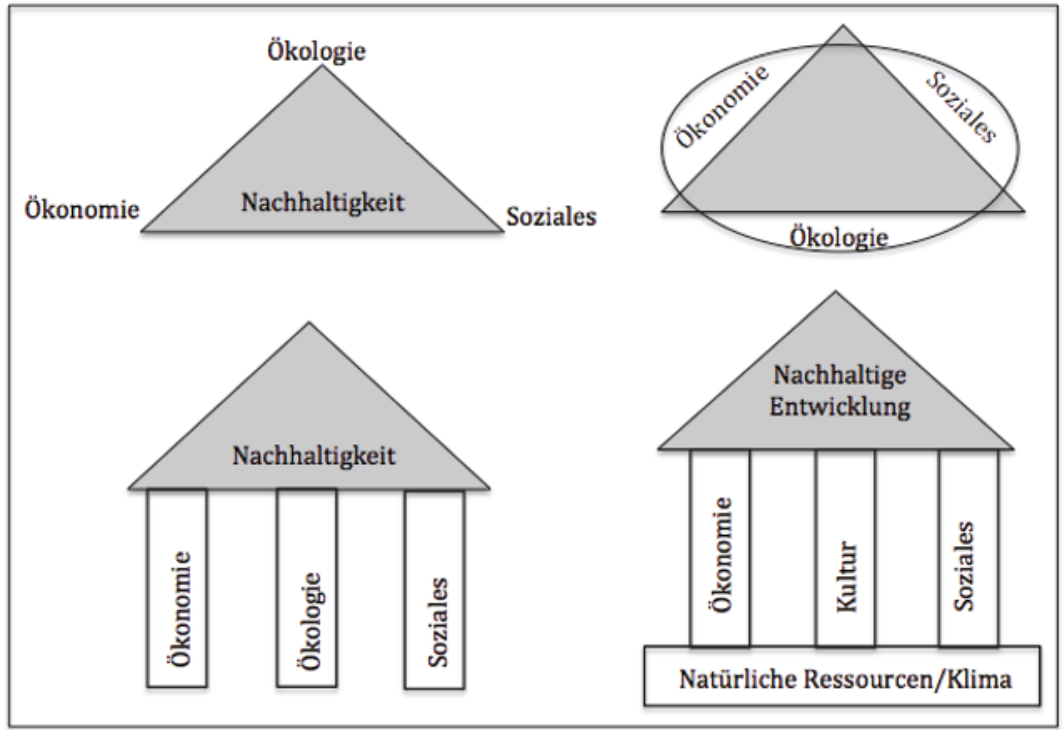

Abb. 1 Die vier Nachhaltigkeitstheorien

Erläuterung und Quellen: oben links: „Nachhaltigkeitsdreieck“ (eigene Darstellung nach Spindler, o. J.); oben rechts: „Magisches Dreieck“ (eigene Darstellung nach Deutscher Bundestag, 1994); unten links: „Drei-Säulen-Modell“ (eigene Darstellung nach Spindler, o. J.); unten rechts: „Gewichtetes Säulenmodell“ (eigene Darstellung nach Stahlmann, 2008) 
Erwartet werden auf Grundlage derartiger Modelle zunächst vor allem Konflikte zwischen den Dimensionen, vernachlässigt werden dagegen Probleme innerhalb. Diese zu überwinden scheint eindeutig nachrangig bewertet zu sein. Am Beispiel der Wasserkraftnutzung zeigt sich aber sehr deutlich, dass gerade diese intra-thematischen Interessenskonflikte mitunter gravierend und besonders schwer zu lösen sein können. Dieser Tatsache tragen komplexere, aber allgemein weniger bekannte Darstellungen Rechnung, wie etwa die „Zauberscheiben der Nachhaltigkeit“ (s. Abbildung 2).

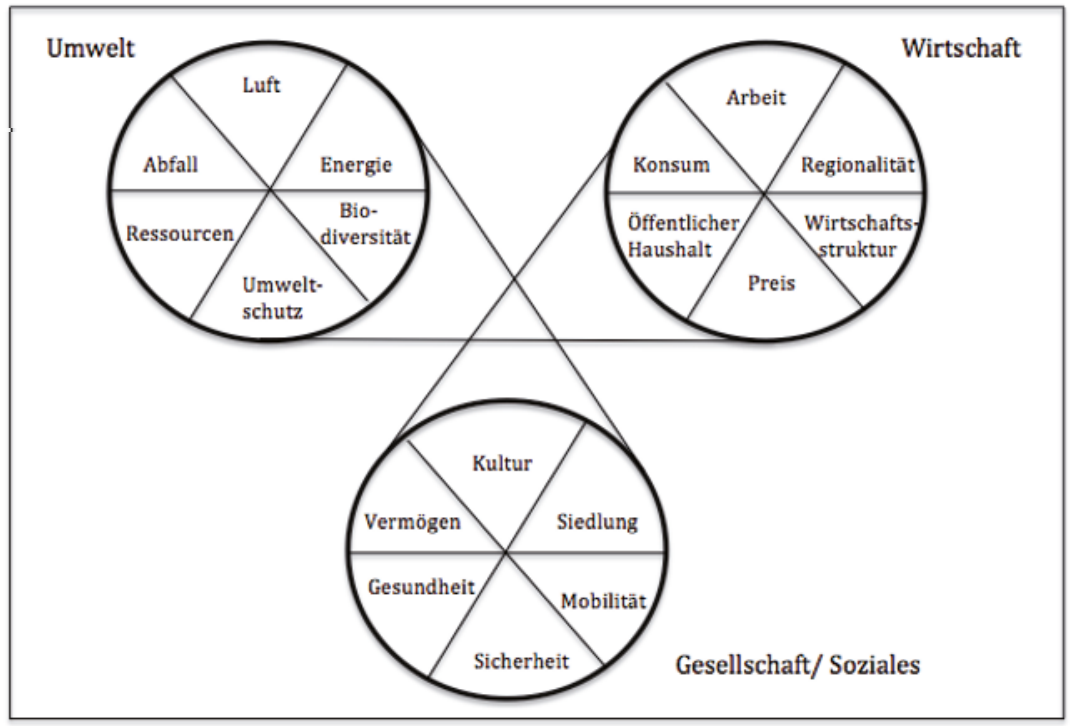

Abb. 2 Zauberscheiben der Nachhaltigkeit

Quelle: Eigene Darstellung nach Diefenbacher, 1997

Dabei stehen Wasserkraftwerke innerhalb der „Umwelt-Zauberscheibe“ im Spannungsfeld zwischen der Nutzung des fließenden Wassers als CO2-neutrale, regenerative und grundlastfähige Energiequelle einerseits und den negativen Auswirkungen auf den Lebensraum aus dem Fluss und seinem natürlichen Überschwemmungsgebiet, der Aue, andererseits. Innerhalb der Dimension der Ökologie existiert folglich ein Interessenskonflikt zwischen dem Klimaschutz und dem Naturschutz, weil für beide Ansätze der gleiche Raum - das Flusssystem - in Anspruch genommen wird, aber konträre Ansprüche vorherrschen. Vor diesem Hintergrund wird in diesem 
Projekt das in Abbildung 3 dargestellte Modell für das Verständnis für Nachhaltige Entwicklung verwendet: Die ökologische Basis, die die Säulen Ökonomie, Kultur und Soziales trägt, steht ihrerseits selbst auf thematischen Säulen. Die Themen, die die Ökologie stützen, lauten Klimaschutz, Ressourcen, Biodiversität und Naturschutz.

\section{Abb. 3}

Erweitertes gewichtetes Nachhaltigkeitsmodell

Quelle: Eigene Darstellung

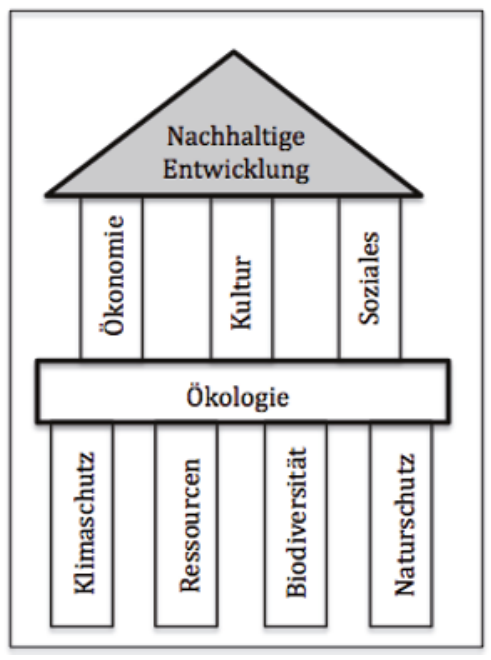

Die Wasserkraftnutzung bedient dabei die Säulen Klimaschutz und Ressourcen, während der Auenschutz dagegen erstrangig wichtig ist für Biodiversität und Naturschutz. Während das fragile Gebilde der Nachhaltigkeit bei einem kompletten Ausfall einer Säule nicht bestehen kann, kann eine Schwächung bis zu einem gewissen Grad noch tolerierbar sein, wenn die restlichen Säulen ausreichend stark sind.

Die Frage, wie die Nutzung der Wasserkraft im Sinne der Nachhaltigkeit $\mathrm{zu}$ bewerten ist, kann folglich damit veranschaulicht werden, ob das erweiterte Nachhaltigkeits-Gebäude stabil bleibt oder ob die Schwächung einzelner Säulen zu einem Einbruch des Gebäudes führt. Dabei ist zunächst unerheblich, wie die weitere Ebene aus Ökonomie, Kultur und Soziales zu bewerten ist.

Folgt man beispielsweise Chasek, David und Welsh Brown (2006) und Diefenbacher et al. (1997), so ist unter nachhaltiger Entwicklung eine Entwicklung zu verstehen, die gleichermaßen im Einklang mit gegenwärtigen wie zukünftigen Bedürfnissen steht. Kann man vor diesem Hintergrund und der geschilderten Problematik aktuell bei der Wasserkraftnutzung von einer nachhaltigen Energie- 
erzeugung sprechen? Wenn die Frage verneint werden muss, stellt sich die Frage, was getan werden kann, um die Pole Klimaschutz und Naturschutz miteinander zu versöhnen - wie Chasek et al. (2006: 425) Nachhaltigkeit in der Umweltpolitik als eine „umweltpolitische Perspektive (verstehen), die die Notwendigkeit betont, gegenwärtige und zukünftige wirtschaftliche Bedürfnisse durch Schutz der Umwelt miteinander zu versöhnen“.

\subsection{Funktion und Bedeutung von Auen}

Die wachsende Beachtung der Auen und der Grund, weshalb dieser Lebensraumtyp mittlerweile in die Nachhaltigkeitsdiskussion mit einbezogen wird, geht auf eine bessere Kenntnis und Anerkennung ihrer Funktion und Bedeutung für den Naturhaushalt und für den Menschen zurück (Bragg, 2000). Diese ist besonders auch vor dem Hintergrund des prognostizierten Klimawandels von größter Bedeutung: als „Hotspots der Artenvielfalt" (Damm et al., 2011:20) beherbergen Auen auf lediglich 6-8\% der Landesfläche Deutschlands circa $2 / 3$ der in Deutschland heimischen Pflanzengesellschaften (Mößmer, 2000), weswegen sie allgemein als Rückgrat der Biodiversität gelten und zu den artenreichsten Ökosystemen in Mitteleuropa gehören (Damm et al., 2011; Dörfer, 2000; Richardson et al., 2007; Stella, 2005; Volk, 2000; Korn et al., 2005; Tockner et al., 2000; Scholz et al., 2009; Gerken et al., 2000).

Der Erhalt der Artenvielfalt ist aus ökologischer Sicht essenziell, da eine hohe genetische Variabilität innerhalb der Arten sowie eine große Artenzahl die Wahrscheinlichkeit erhöhen, dass eine Anpassung an sich ändernde Umweltbedingungen erfolgen kann und frei werdende ökologische Nischen innerhalb von Ökosystemen durch funktionell ähnliche Arten besetzt werden können (BMU, 2007). Zudem sind Auen nicht nur direktes Habitat vieler Arten, sondern fungieren als Korridor für den genetischen Austausch (Damm et al., 2011; Detering, 2000; Pfadenhauer, 1997). Prognosen zufolge ist bis 2100 mit einer mittleren Temperaturerhöhung von 1,8 bis $4,0^{\circ} \mathrm{C}$ zu rechnen (IPCC, 2007). Wiederkehrende ausgeprägte Hitzesommer einerseits und beispielsweise auch die Häufung extremer Niederschlagsereignisse gelten als wahrscheinliche Szenarien für Mitteleuropa (Frei et al., 2006; Kramer et al., 2008; Rowell \& Jones, 2006). So drohen während der Sommermonate gleichermaßen mehr Trockenperioden und extreme Flutereignisse (Kunstmann et al., 2004). In diesem Zusammenhang ist von großer Bedeutung, dass Auen durch die Bereitstellung von Retentionsräumen Extremereignisse wie Hoch- und Niedrigwasserereignisse abmildern sowie einen lokalen Ausgleich des Klimas bewirken (Damm et al., 2011; Debano \& Schmidt, 1990; Ellenberg \& Leuschner, 2010; Korn et al., 2005; Scholz et al., 2009): Da Auen im Hochwasserfall Teil des Abflussgeschehens werden, können 
zum einen höhere Abflussmengen abgeführt werden und gleichzeitig ermöglicht die Zwischenspeicherung in der Fläche ein Abbremsen der Hochwasserwelle und einen verminderten Scheitelabfluss, der auf einen längeren Zeitraum gestreckt wird. Durch die verzögerte Wasserrückführung aus den Oberflächenwasserspeichern der Aue werden somit Hochwasserspitzen reduziert und gleichzeitig wird ein positiver Einfluss in Niedrigwasserzeiten erreicht (Korn et al., 2005).

Zudem wirken sich Auen auch positiv auf die Grundwasseranreicherung sowie -reinigung aus (Damm et al., 2011; Debano \& Schmidt, 1990). Auch ein lokaler Ausgleich des Klimas durch Auen (Damm et al., 2011; Debano \& Schmidt, 1990; Ellenberg \& Leuschner, 2010; Korn et al., 2005; Scholz et al., 2009) hilft die Folgen einer Klimaerwärmung abzumildern. Da große Mengen von Kohlendioxid in den Auenböden und im Holz gespeichert werden (Augustin, 2001; Stella, 2005), sind Auen als eine bedeutende Stoffsenke zu betrachten, die der Steigerung von Kohlendioxid in der Atmosphäre aktiv entgegenwirkt (Korn et al., 2005; Richardson et al., 2007; Alldredge \& Moore, 2012). Der Lebensraumtyp der Weichholzaue, der in diesem Projekt Thema ist, hat im System aus Fluss und Aue eine besonders bedeutende Rolle inne. Die dort bestimmenden Pionier-Baumarten wirken selbst gestaltend auf den Fluss ein, indem sie die herrschenden morphodynamischen Prozesse beeinflussen (Pasquale et al., 2012), da sie schon bald nach ihrer Etablierung substratstabilisierendes Wurzelwerk bereitstellen (Stella, 2005). Durch das schnelle Wachstum der Weichhölzer wird zudem klimawirksames Kohlenstoffdioxid fixiert und zeitnah eine erste vertikale Zonierung erreicht (Stella, 2005).

\subsection{Situation der Auen}

Als traditionelle Siedlungsräume des Menschen wurden Auen in Mitteleuropa bereits seit der Steinzeit besiedelt (Scholten et al., 2005). Während seit dem frühen Mittelalter zunächst der terrestrische Teil der Auen durch Rodungen anthropogen überprägt wurde (Ellenberg \& Leuschner, 2010; Scholz et al., 2009), erfolgte ab dem 12. Jahrhundert bereits der Bau erster Deiche. Seit dem frühen 19. Jahrhundert begann letztlich der systematische Ausbau der Fließgewässer mit dem Ziel die Auen großflächig nutzbar zu machen (Scholz et al., 2009). Deichlinien wurden geschlossen und erhöht, bisher mäandrierende oder verzweigte Flüsse begradigt, Entwässerungsmaßnahmen durchgeführt und die Auenwälder weiter gerodet (Foeckler \& Bohle, 1991; Gerken, 1988; Scholten et al., 2005). Im 20. Jahrhundert erfolgte schließlich noch der Bau von Staustufen.

Diese diversen anthropogenen Eingriffe (Richardson et al., 2007) haben zu einem starken Rückgang der Auen geführt. Nach Brunotte et al. (2009) sind in Deutschland 
lediglich $1 / 3$ der ehemaligen Überschwemmungsflächen heute überhaupt noch bei Hochwassersituationen überflutbar. In dieser so genannten „rezenten Aue" befinden sich aber zusätzlich $90 \%$ in einem deutlich, stark oder sehr stark veränderten Zustand, während die Kategorie „sehr gering verändert“ weniger als ein Prozent ausmacht. So ist auch der Zustand des Lebensraumtyps der „Auwälder" nach dem Bericht Deutschlands an die Europäische Kommission zum Zustand der biologischen Vielfalt als ungünstig zu bezeichnen. In Deutschland sind, wie in Riecken et al. (2006) geschildert wird, bereits $83 \%$ aller Biotoptypen der Flüsse und Auen gefährdet. Für Bayern geben Wenger et al. bereits 1990 einen Verlust von $80 \%$ der Auwälder mit der Perspektive, dass aufgrund der konkurrierenden Interessen weitere große Gebiete zerstört werden, an. Naturnahe Auen gehören damit zu den am stärksten gefährdeten Lebensräumen in Europa (Damm et al., 2011; Korn et al., 2005; Mosner et al., 2008). Besonders gut erforscht und dokumentiert ist der Verlust von Auwaldflächen auch in den semi-ariden Bereichen im westlichen Nordamerika (Mahoney \& Rood, 1998; Rood, 2000).

Die Gefährdung gilt in besonderem Maße für die in diesem Projekt betrachteten Weichholzauen. Die Einschätzung von Wenger et al. aus dem Jahr 1990, dass Weichholzauwälder fast vollständig verschwunden sind, wird auch in neueren Quellen wie Walentowski et al. (2004), Sautter (2003) und Schubert et al. (2010) bestätigt.

Auch an der Donau und ihren alpinen Zuflüssen ist der Zustand der Auen sehr stark durch den Flussausbau in Kombination mit einer intensiven Wasserkraftnutzung geprägt. Die wenigen als gering verändert eingestuften Abschnitte liegen an der Donau im Bereich von Engtälern mit Auenbreiten von maximal 500 Metern sowie stromabwärts der Isarmündung (Brunotte et al., 2009). Insgesamt bilanzieren Brunotte et al. (2009), dass von 1111 Quadratkilometer morphologischer Aue nur noch rund 290 Quadratkilometer als rezente Aue überflutbar sind.

In der Nationalen Strategie zur biologischen Vielfalt (BMU, 2007) wird aufgrund der extremen Gefährdungssituation als konkretes Ziel bis 2020 angeführt, dass „Fließgewässer und ihre Auen in ihrer Funktion als Lebensraum soweit gesichert [sind], dass eine für Deutschland naturraumtypische Vielfalt gewährleistet ist" (BMU, 2007: 35). Hierfür soll angestrebt werden, dass die „Nutzung der Wasserkraft bei Modernisierung oder Neubau der Wasserkraftanlage unter Beibehaltung der charakteristischen Eigenarten des Fließgewässers, der Gewährleistung der ökologischen Durchgängigkeit sowie der Verbesserung oder Wiederherstellung der Funktionsfähigkeit" (BMU, 2007: 36) erfolgt. Die bisher umgesetzte Technik beim Bau von Wasserkraftwerken erfüllt diese Forderung jedoch nicht und es sind zahlreiche negative Auswirkungen von Wasserkraftanlagen dokumentiert (exemplarisch: Brunotte et al., 2009; Müller et al., 2006). Dadurch, dass die Wasserkraft aber als weitgehend emissionsfreie und regenerative Energiequelle gilt, ist ihre 
Nutzung ein essentieller Bestandteil der Konzepte zur Energieproblematik und zum Klimawandel (BMWI, 2012; Müller et al., 2006).

In Bayern ist die Wasserkraft mit einem Anteil von etwa $60 \%$ die bedeutendste und am intensivsten ausgebaute Form der Stromerzeugung aus regenerativen Energiequellen mit langer Tradition. Zur Umsetzung der Energiewende heißt es im Bayerischen Energiekonzept „Energie innovativ“, dass die noch vorhandenen Wasserkraftpotenziale verstärkt genutzt werden sollen. Hierzu müsse die Wasserkraft „schnell, konsequent und umweltverträglich ausgebaut werden“" (StMWI, 2011: 9). So soll die Stromerzeugung aus Wasserkraft (ohne Pumpspeicherkraftwerke) in Bayern bis 2021 um rund 2 Milliarden Kilowattstunden pro Jahr erhöht werden, was bedeutet, dass die derzeitige Erzeugung von durchschnittlich rund 12,5 Milliarden Kilowattstunden pro Jahr damit um gut $15 \%$ auf rund 14,5 Milliarden Kilowattstunden pro Jahr gesteigert werden würde. Die für die Zulassung der Wasserkraftnutzung zuständigen Behörden werden durch eine klare politische Zielfestlegung zugunsten einer verstärkten Wasserkraftnutzung einschließlich des umwelt- und naturverträglichen Neubaus von Wasserkraftwerken gestützt. Diese Zielrichtung gilt damit auch für die letzten freifließenden Bereiche der Donau mit Auswirkungen für die Auwaldlebensräume. Der aktuell noch frei fließende Donauabschnitt zwischen Straubing und Vilshofen (Flusskilometer 2330-2248) weckt zudem Begehrlichkeiten seitens der kommerziellen Schifffahrt, weshalb hier eine umfassende Studie über ausbaubedingte Veränderungen durch die zur Diskussion stehenden Ausbauvarianten auf die Fläche erarbeitet wurde (Peper et al., 2012). Vor diesem Hintergrund besteht dringender Forschungsbedarf, um die Auswirkungen von weiteren Wasserkraftanlagen auf den Lebensraumtyp der Weichholzaue besser abschätzen zu können und gegebenenfalls stichhaltige Argumente zu liefern, wieso von einem weiteren Ausbau abgesehen werden muss.

\subsection{Bestimmende Faktoren des Vorkommens und der Gefährdung von Weichholzauen}

Natürliche Auen sind geprägt durch ständige Wasserstandswechsel im Fluss und der angrenzenden Aue (Korn et al., 2005; Scholz et al., 2004), die zu einem permanenten Wechsel von Überflutung und Trockenfallen führen (Brunotte et al., 2009; Damm et al., 2011; Dister, 1991; Gerken, 1988; Henrichfreise, 2000; Pfadenhauer, 1997; Robinson, 2000; Scholz et al., 2009; Schwartz, 2001). Ökologisch intakte Auen zeigen daher eine große Dynamik bezüglich Wasser und Sediment und unterliegen räumlich und zeitlich ständigen Veränderungen (Dister, 1998; Hejda \& Pyšek, 2006). Diese Dynamik ermöglicht die Koexistenz verschiedenster speziell 
angepasster und gleichzeitig auf sie angewiesener Lebensraumtypen auf engem Raum (Dörfer, 2000; Gerken et al., 2000). Der Weichholzauwald schließt dabei flussseits an den Hartholzauwald an.

Die Bundesanstalt für Gewässerkunde hat für Weichholzauenstandorte an der Donau im Bereich der Isarmündung auf Grundlage umfangreicher Untersuchungen modelliert, dass der Lebensraumtyp der Weichholzaue im Bezug auf den Grundwasser-Flurabstand ein Standortpotenzial zwischen $-20 \mathrm{~cm}$ und $70 \mathrm{~cm}$ besitzt (Peper et al., 2012). Dadurch, dass die Bestände meist nur wenig über dem Mittelwasser-Stand stocken, unterliegt die Weichholzaue als typisches „by natural disturbance driven ecosystem“ (Walentowski et al., 2004) ständigen Umgestaltungen und es herrschen extreme Standortverhältnisse bezüglich Bodenwasser- und Lufthaushalt vor (Walentowski et al., 2004). Kennzeichnend sind periodische und langandauernde Überflutungen und wenig entwickelte Böden aus frisch angelandeten Flusssedimenten mit unterschiedlichen Korngrößen, die aber meist kalk- und nährstoffreich sind (Rambla-Kalkpaternia, Gley-Paternia oder Paternia-Gley). Allgemein kann man zwischen einer dynamischen Weichholzaue und einer nassen Weichholzaue unterscheiden. Während sich erstere durch frische Sedimentation, hohe Wasserstandsschwankungen, circa 100-200 Tage Überflutung pro Jahr und fehlenden Grundwasserstand auszeichnet, befindet sich die nasse Weichholzaue flussferner beispielsweise in nassen Mulden. Diese Standorte zeichnen sich durch geringere mechanische Belastungen und einen Anschluss an das Grundwasser aus.

Alle typischen Gehölzgesellschaften des Lebensraumtyps der Weichholzaue in Deutschland gehören der Klasse Salicetea purpurea (Uferweidengebüsche und Weidenwälder) (Sautter, 2003; Schubert et al., 2001 nach Moor, 1958) an. Die einzigen Charakterarten dieser Klasse sind Weiden (Salix purpurea, Salix alba, Salix fragilis, Salix viminalis) und die Schwarzpappel (Populus nigra) (Oberdorfer, 1992; Scholz et al., 2004; Schubert et al., 2001). Diesen Arten ist ihre Spezialisierung auf Pionierstandorte mit häufigen und intensiven (fluvialen) Störungen gemeinsam (Oberdorfer, 1992; Richardson et al., 2007; Walentowski et al., 2004). So benötigen sie als Nacktbodenkeimer aufgrund ihrer Konkurrenzschwäche und Lichtbedürftigkeit zur Keimung möglichst vegetationsfreien, offenen und feuchten Boden. Nach einer sowohl gegen Trocken- als auch gegen Überstauungsstress vulnerablen Phase ertragen sie deutlich längere Überflutungszeiten als Konkurrenzarten wie Eschen, Eichen oder Ahornarten (Dister, 1983; Hughes et al., 2001; Richardson et al., 2007; Sautter, 2003; Scholz et al., 2004; Schubert et al., 2001).

Als Ursache für den Rückgang der Weichholzauen wird im wissenschaftlichen Diskurs in erster Linie die mangelnde morphogenetische Dynamik angeführt, weil deshalb keine oder kaum Pionierstandorte entstehen können. Diese aber sind - wie oben beschrieben - essenziell für die erfolgreiche Verjüngung von Weiden 
und Pappeln (Ellenberg \& Leuschner, 2010; Müller et al., 2006; Richardson et al., 2007; Scholz et al., 2004).

Diese mangelnde Dynamik geht zum einen auf die weitreichenden Begradigungen sowie Eindeichungen der Flüsse und zum anderen auf das Abflussmanagement durch Staubauwerke zurück, die bei der aktuell umgesetzten Technik zur Gewinnung von Hydroenergie notwendig sind: Durch die Laufverkürzung und die gleichzeitige seitliche Fixierung der Flüsse kam es zu verstärkter Tiefenerosion und damit zur Tieferlegung der Sohle und des anschließenden Grundwasserspiegels. Der Donauverlauf zwischen Neuburg und Ingolstadt wurde beispielsweise im Zeitraum von 1826 bis 1926 um 17\% verkürzt, wodurch sich das Fließgefälle von 0,058 auf durchschnittlich 0,068\% erhöhte (Margraf, 2004 nach Stammer, 1954). Die von ursprünglich 5 Kilometer Breite auf 120 Meter eingeengte Donau zeigte folglich die beschriebene Tendenz zur Tiefenerosion, die durch den Bau der Staustufen im Oberlauf und an den Zuflüssen über den Mangel an Geschiebefracht noch verschärfte. Folge ist, dass im genannten Bereich das Flussbett zu Beginn der Regulierung um 1800 cira 3 Meter höher lag, als heute (Margraf, 2004). Das früher einheitlich verlaufende Fließgefälle zeigt nach dem Bau der Staukette zwischen der Lechmündung und Vohburg die für Stauketten typische treppenartige Ausprägung mit stark erniedrigtem Gefälle oberhalb und einem erhöhten Gefälle unterhalb der Querbauwerke. Die Fließgewässergerinne selbst wurden durch die Ausbaumaßnahmen insgesamt deutlich leistungsfähiger, weshalb kleinere Hochwasserereignisse seither innerhalb des direkten Flussbetts verbleiben und keine Überflutungen des Umlandes mehr hervorrufen. Statt dem nötigen "flood-pulse“ tritt hier lediglich der in humiden Bereichen weitaus weniger wirksame „flow-pulse“ auf (Tockner et al., 2000). Aufgrund der fast lückenlos errichteten Staubauwerke ist es zudem möglich, den Abfluss der Flüsse - abgesehen von Extremereignissen - gezielt beeinflussen zu können. Kleinere Hochwässer werden in den Stauseen abgefangen und nachfolgend kontrolliert abgelassen. Auf diese Weise wird zum einen ein Beitrag zum Hochwasserschutz geleistet und zum anderen die Fallhöhe des Wassers innerhalb der Turbinen möglichst konstant gehalten. Gerade diese Konstanz steht im Gegensatz zum Bedarf der Auenlebensräume an dynamischen Abflussverhältnissen (Bunn \& Arthington, 2002; Ellenberg \& Leuschner, 2010; Jürging \& Patt, 2005; Margraf, 2004; Sautter, 2003; Zahlheimer, 1979).

Intensiviert wird die Problematik im flussabwärts von Staubauwerken gelegenen Flussabschnitt durch die mangelnde Durchgängigkeit der Staustufen. Während die negativen Auswirkungen auf wandernde Fischarten bereits seit langem erkannt sind, erhält die Barrierewirkung auf das Sediment erst seit relativ kurzer Zeit die nötige Beachtung. Da oberhalb der Staubauwerke die Fließgeschwindigkeit des Flusses auf ein Minimum reduziert wird, sinkt gleichzeitig auch die Transport- 
kraft des Wassers. Folglich werden die bisher transportierten Partikel im Stausee abgelagert. Dieser Prozess führt im flussabwärts gelegenen, ungestauten und damit fließenden Flussabschnitt mit wieder erhöhter Transportkraft zu einem Transportdefizit. Infolgedessen wird dort bisher abgelagertes Material wieder mobilisiert und entsprechend der Fließrichtung wegtransportiert. Da auch hier der seitliche Uferbereich des Flusses fixiert ist, konzentriert sich die überschüssige Energie ausschließlich auf die Gewässersohle und verschärft die Problematik der Tiefenerosion im Unterwasserbereich von Staustufen deutlich (Müller et al., 2006). Das Niveau der ehemaligen Auenflächen ähnelt größtenteils eher einer neuen Niederterrasse des Flusses und hat folglich fast jeglichen Kontakt zur hydrologischen Dynamik des Flusses verloren. Zusätzlich wird die Zusammensetzung der Flussfracht durch die Staubauwerke modifiziert, da das grobe Material aus den Alpen bereits in Stauseen der Zuflüsse zurückgehalten wird und dadurch nicht mehr als Transportmaterial in der Donau zur Verfügung steht. Diese Tatsache verändert automatisch auch die Korngrößenzusammensetzung später abgelagerter Rohbodenstandorte hin zu feinerem Material (Cyffka \& Haas, 2008), das für Hartholzauen typisch ist. Die Behinderung der Abwärtsdrift für Diasporen wird für die Klassenkennarten der Weichholzaue als nachrangig eingestuft, weil die extrem leichten und kleinen Samen von Weiden und Pappeln zum einen größtenteils durch Anemochorie verbreitet werden und zum anderen für erfolgreiche Verjüngung auf Standorten in passenden Höhenverhältnissen ein vorangegangenes Hochwasserereignis notwendig ist. Hochwasserereignisse führen ihrerseits gewöhnlich zu einer Öffnung der Stauwehre, wodurch auch im unmittelbaren Staubereich Abfluss vorherrscht und keine Ablagerung der Flussfracht im Staubereich stattfindet.

Der Einfluss des Klimas auf den Lebensraumtyp der Weichholzaue darf dagegen nicht vernachlässigt werden: gerade Weiden sind für eine erfolgreiche Verjüngung darauf angewiesen, dass Hochwässer vor der Reifung ihrer Früchte auftreten. Das hydrologische Regime hängt dabei seinerseits von den klimatischen Bedingungen ab. Bleiben Hochwasserereignisse vor der Samenabgabe aus, stehen keine passenden Keimhabitate zur Verfügung, weil zum einen keine fluvial bedingten Rohbodenstandorte entstehen konnten oder zum anderen die etablierte Vegetation auf bereits vorhandenen Flächen nicht ausreichend geschädigt wird, um nötige Freiflächen zu erzeugen. Tritt dagegen zusätzlich zu frühen Hochwässern ein weiteres spätes Überflutungsereignis nach der Weidenkeimung auf, ist mit sehr hohen Ausfällen der Keimlinge zu rechnen, da die weidenspezifische hohe Überflutungstoleranz erst bei etablierten Bäumen festzustellen ist. Die Keimlinge dagegen sind vulnerabel gegenüber Überflutung und gegen Trockenstress (Ellenberg \& Leuschner, 2010; Richardson et al., 2007). Nachdem Weidensamen durchschnittlich lediglich kurze Zeit keimfähig sind ist eine erfolgreiche Weidenverjüngung nur möglich, 
wenn das hydrologische Regime - seinerseits gesteuert durch das Klima - genau zu dieser Zeit die passenden Verhältnisse geschaffen hat (Sautter, 2003). Im Zuge des prognostizierten Klimawandels wird von diversen Veränderungen der klimatischen Bedingungen ausgegangen. Es muss in Betracht gezogen werden, dass die Verjüngung der Weiden somit von den sich wandelnden klimatischen Bedingungen beeinträchtigt wird. Da es speziell bezogen auf diesen Einfluss noch keine publizierten Studien gibt, wird im Zuge des Projekts mittels Dendrochronologie und Zeitreihenanalysen untersucht, inwieweit sich das Klima und das hydrologische Regime in Zeiten mit erfolgreicher natürlicher Verjüngung der Charakterarten von den Bedingungen unterscheiden, die ein Scheitern bedingen.

Die Auswirkungen einer durch Neophyten veränderten Konkurrenzsituation auf die Keimungs- und Etablierungschancen von Charakterarten der Weichholzauenbestände wurde im wissenschaftlichen Diskurs bisher nicht tiefergehend behandelt. Es gibt lediglich Studien, die allgemein den Artenreichtum in Auenstandorten mit und ohne Neophytenvorkommen vergleichen (Hejda \& Pyšek, 2006; Hejda et al., 2009; Lambdon et al., 2008). In diesem Forschungsprojekt wird davon ausgegangen, dass es sich hierbei aber um einen durchaus bedeutsamen Faktor bei der Verjüngung der Charakterarten der Weichholzauwäldern handeln kann, dem weiter nachzugehen ist.

Daneben werden im Allgemeinen auch die Vernichtung von Sämlingsanflug durch Wellenschlag, Uferbeweidung sowie die anthropogene Veränderung der Geschlechterverteilung in den Weiden- und Pappelpopulationen als wichtige Faktoren betrachtet (Scholz et al., 2004), wobei die Auswirkungen von Wellenschlag und Beweidung in diesem Projekt aufgrund der lokalen Gegebenheiten vernachlässigt werden können.

\section{$2 \quad$ Herangehensweise im Projekt}

Das Projekt beschäftigt sich speziell mit den Auswirkungen der Wasserkraftnutzung auf den Lebensraumtyp der Weichholzaue, der durch die intensive Nutzung von Fluss und Aue als hochgradig bedroht gilt (Oberdorfer, 1992; Riecken et al., 2006; Sautter, 2003; Walentowski et al., 2004; Wenger et al., 1990) und infolgedessen als prioritärer Lebensraum entsprechend der EU-FFH Richtlinie geschützt ist (BayLfu und BayLWF, 2004; Walentowski et al., 2004).

Das zentrale übergeordnete Ziel des Forschungsprojekts ist es, ein Konzept zur ökologischen Regenerierung und Sicherung für Weichholzauenbestände im Umfeld von Wasserkraftanlagen zu erarbeiten, wodurch der Erhalt, besonders aber 
auch die neue Etablierung von Weichholzauenbeständen in deren Einflussbereich wieder ermöglicht werden soll. Zu diesem Zweck gilt es zunächst den Zustand der aktuell noch vorhandenen Weichholzauenbestände zu ermitteln. Danach müssen die Steuerfaktoren ausfindig gemacht werden, die für den aktuellen Entwicklungszustand der wenigen Restbestände an Weichholzauen verantwortlich sind. Mit diesem Wissen sollen letztlich insbesondere die Chancen ermittelt werden, die sich beispielsweise durch eine entsprechende Steuerung der Stauanlagen oder Gestaltung des Umlandes der Wasserkraftanlagen ergeben können, um die Situation in deren Umfeld deutlich zu verbessern (s. Abbildung 4).

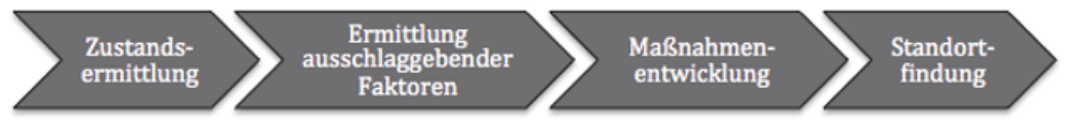

Abb. 4 Schematische Darstellung des Projektaufbaus

Quelle: Eigene Darstellung

Das in diesem Beitrag behandelte Projekt befindet sich derzeit noch in der Umsetzungsphase, weshalb an dieser Stelle noch keine Ergebnisse diskutiert werden können. Aus diesem Grund wird sich auf eine Erläuterung der theoretischen Basis zur Ermittlung der ausschlaggebenden Faktoren sowie Maßnahmenentwicklung konzentriert.

\section{Forschungsansätze zur Verbesserung der Situation der Weichholzauenbestände}

\subsection{Ermittlung des aktuellen Zustands von Weichholzauenbeständen an der bayerischen Donau im Umfeld von Wasserkraftwerken}

Wie unter 1.4 bereits erläutert, sind die einzigen Charakterarten der im Projekt betrachteten Klasse Salicetea purpurea Weiden (Salix purpurea, Salix alba, Salix fragilis, Salix viminalis) und die Schwarzpappel (Populus nigra) (Oberdorfer, 1992; Scholz et al., 2004; Schubert et al., 2001). Bezogen auf die Krautvegetation kann eine Vielzahl an Arten vertreten sein, die mitunter auch Charakter- und Kenn- 
arten anderer Pflanzengesellschaften sind. Besonders häufig sind hygronitrophile Hochstauden (Oberdorfer, 1992; Sautter, 2003; Scholz et al., 2004). Als stete Begleiter gelten nach Schubert et al. (2001) das Kletten-Labkraut (Galium aparine), das Rohrglanzgras (Phalaris aruninacea), die bereifte Brombeere (Rubus caesius) sowie die große Brennnessel (Urtica dioica). Ausschlaggebend ist, dass die Kombination der vorkommenden Arten keiner der typischen Waldgesellschaften zugeordnet werden kann.

Pflanzensoziologisch lässt sich der Zustand einer Weichholzaue folglich daran messen, ob

1. die Klassencharakterarten in einem günstigen Entwicklungszustand sind und ob 2. die Krautvegetation eine Tendenz hin zu einer anderen Pflanzengesellschaft zeigt.

Der Zustand der Klassencharakterarten kann zum einen an der Stetigkeit und Häufigkeit ihres Vorkommens und zum anderen daran bemessen werden, ob Verjüngung gelingt oder der Bestand überaltert. Auch die Ausgewogenheit der Geschlechterverteilung ist für die diözischen Weiden essenziell. Diese kann während der Blüte aufgrund des unterschiedlichen Phänotyps der Blüten leicht beurteilt werden. Die Krautvegetation wird über pflanzensoziologische Analysen entsprechend der Methode von Braun-Blanquet (1964) überprüft. Anschließend werden zur Charakterisierung der Pflanzengesellschaften die absoluten Artenzahlen der Flächen, der Shannon Diversity Index H, der Evenness Index sowie die Ellenberg Zeigerwerte ermittelt und analysiert. Zusätzlich erfolgen indirekte Ordinationsverfahren (Detrended correspondence analysis) und Clusteranalysen, bei denen auch die abiotischen Parameter einfließen.

Als relevante abiotische Parameter zur Charakterisierung von Weichholzauen gelten die Korngrößenzusammensetzung, die Nährstoffbedingungen und der $\mathrm{pH}$ Wert der Böden sowie hydrologische Kenngrößen. Ein Verständnis der hydrologischen Situation in den Projektgebieten kann über Pegeldaten entwickelt werden. Von besonderer Bedeutung ist dabei aktuelle Daten und Daten des Zeitraums vor und nach Staustufenbau zu akquirieren: Wenngleich Henrichfreise (2000) die Verwendung von Grundwassermodellen in Auen grundsätzlich ablehnt, weil der heterogene Bodenaufbau das ungehinderte Durchdringen des Grundwassers durch den Boden verhindert, müssen diese weiterhin Verwendung finden, da keine Alternative existiert. In der Studie der Bundesanstalt für Gewässerkunde (Peper et al., 2012) wurden aus einer Reihe von potenziellen Einflussfaktoren (darunter auch Bodenparameter) mit Hilfe diverser statistischer Verfahren (logarithmische HOF-Modelle, Nichtmetrische Multidimensionale Skalierung, Spearman-Rang-Korrelationstest, Klassifikationsbäume) die Parameter "Grundwasser-Schwankungen“ 
und „Grundwasser-Flurabstand“ als meist-differenzierende Faktoren der Vegetation herausgearbeitet.

\subsection{Förderung der Keimungs- und Etablierungsbedingungen von Silberweidenbeständen}

Dendrochronologische Untersuchungen sollen Aufschluss darüber geben, wann sich die letzten größeren Silberweidenbestände im Untersuchungsgebiet ober- und unterhalb von Staustufen etablieren konnten. Durch Kenntnis des Keimungsjahres können die zugehörigen klimatischen und hydrologischen Kenngrößen ermittelt werden, die eine natürliche Etablierung ermöglicht haben. Angelehnt an die intensiven Forschungsarbeiten im semi-ariden Nordwesten Amerikas durch Mahoney und Rood (1998), Rood (2000), Rood et al. (2003) und Stella et al. (2010) soll durch die Analyse der hydrologischen und klimatischen Daten ein Muster herausgearbeitet werden, bei dessen Erfüllung in einer definierten Höhenlage zum Flusspegel Weidenverjüngung erfolgen kann. Die Erkenntnisse in den USA mündeten im so genannten "Recruitment-Box“-Modell, das im Zuge des Projektes für die Silberweidenbestände an der deutschen Donau, einem Raum mit humidem Klima, erarbeitet werden soll: Dieses quantitative und integrative Modell zeigt die Beziehung zwischen hydrologischen Parametern und dem Keimungserfolg von Auwaldpflanzen (besonders Pappeln und Weiden) in semiariden Bereichen auf (Rood 2000). Entscheidend ist zum einen die Höhenlage für eine erfolgreichen Pappelverjüngung (s. Abbildung 5). Die Obergrenze wird in den semiariden Gebieten durch Trockenstress festgelegt. Keimen die Pflanzen oberhalb, verlieren sie den Anschluss an den „Capillary fringe“, erfolgt die Keimung unterhalb, droht in den Folgejahren Schädigung durch künftige Überflutungen (Mahoney \& Rood, 1998; Scott et al., 1996). 


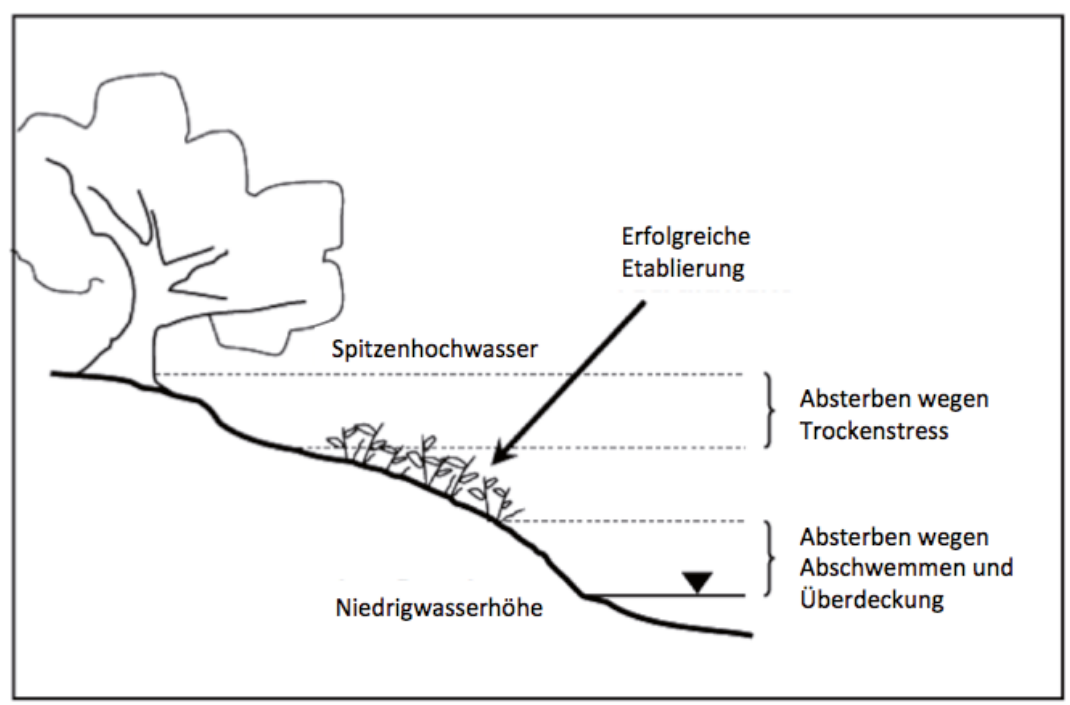

Abb. 5 Etablierung von Keimlingen von Weichhölzern

Erläuterung: Typisches Muster der Etablierung von Keimlingen von Weichhölzern entlang von semiariden alluvialen Flusssystemen. Übertragbarkeit auf Flusssysteme in humiden Klimaten ist größtenteils möglich, da auch hier bei Kontaktverlust der Wurzeln zum "Capillary Fringe“ (Kapillarsaum) mit einer hohen Mortalität aufgrund von Trockenstress gerechnet werden muss.

Quelle: Eigene, veränderte Darstellung nach Stillwater Sciences, 2006

Der zweite entscheidende Parameter ist das hydrologische Regime (s. Abbildung 6): Entscheidend ist der Zeitpunkt des Hochwasserereignisses sowie das nachfolgende Absinken des Wasserspiegels. Im intensiv untersuchten semi-ariden Nordwesten Amerikas ist ein Hochwasserereignis vor der Aussamung der Pappeln notwendig, um die vorhandene Vegetation zu schwächen. Zum Zeitpunkt der Samenabgabe muss dann ein Wasserstandsrückgang einsetzen, so dass wassergesättigte und vegetationsfreie Keimbettstrukturen freigelegt werden. Der zu Beginn schnelle Wasserstandsrückgang muss sich in der Folge dann entsprechend verlangsamen, damit die Wurzeln der Jungpflanzen den Kontakt zum "Capillary Fringe“ nicht verlieren. Durch das Absterben von - im Vergleich zum Flusspegel - zu hoch beziehungsweise zu tief gekeimten Pflanzen entsteht die typische Bänderung der Weichholzauen. 


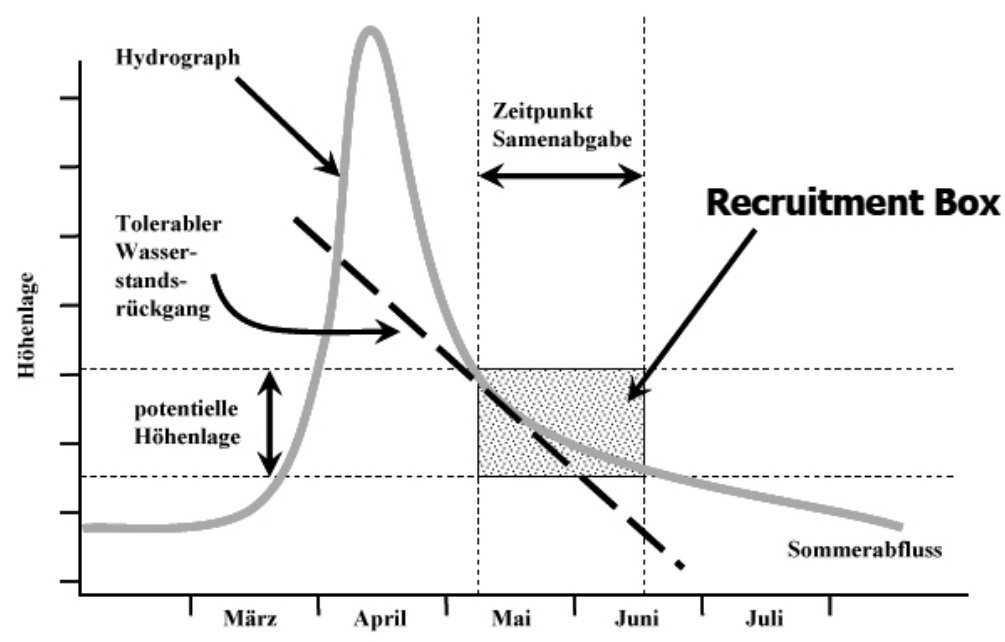

Abb. 6 Recruitment Box

Schematische Darstellung der Recruitment Box, die sich aus einer Verschneidung der Höhenlage (y-Achse) mit der Zeit (x-Achse), der Periode der Samenabgabe und dem Hydrographen ergibt.

Quelle: Veränderte Darstellung nach Stillwater Sciences, 2006

Ebenso wie im semiariden Raum sind in humiden Klimaten vor der Samenabgabe der Weiden und Pappeln Hochwasserereignisse notwendig, um Rohbodenstandorte oder zumindest vegetationsfreie Standorte zu schaffen. Auch ist gleichermaßen nach der Aussamung ein Rückgang des Flusspegels nötig um die Keimhabitate freizulegen. Fraglich ist allerdings, ob die nachfolgende Rate an Wasserstandsrückgang essentiell ist oder ob der Wasserbedarf durch Niederschläge gedeckt werden kann. Es wird davon ausgegangen, dass in humiden Bereichen besonders auch das Ausbleiben von Hochwasserereignissen nach der Keimung ausschlaggebend ist. Ziel der Arbeiten ist es, ein Recruitment-Box Modell für die Verjüngung von Weiden und Pappeln im humiden Raum zu erarbeiten.

Im Zuge der Analyse der Pegel- und Klimadaten werden nicht nur die konkret im Keimungsjahr vorherrschenden Bedingungen erfasst. Zeitreihenanalysen geben Aufschluss über die Frage, ob die seither ausbleibende Weidenverjüngung darauf zurückzuführen sein kann, dass keine passende Kombination der hydrologischen Verhältnisse mehr aufgetreten ist. Durch die Kombination der hydrologischen Da- 
ten mit den Klimadaten wird nachfolgend überprüft, ob gewöhnliche klimatische Schwankungen, der anthropogene Klimawandel oder die Steuerung des Abflusses durch Staustufen dafür verantwortlich sind.

\subsection{Bestimmung der Bedeutung der Konkurrenz von Neophyten}

Auenökosysteme - insbesondere mit steigender anthropogener Überprägung (Décamps et al., 1995; Richardson et al., 2007; Tockner et al., 2001) - gelten als besonders anfällig für die Einwanderung invasiver Neophyten (Hood \& Naiman, 2000; Hulme \& Bremner, 2006; Kasperek, 2004; Pyšek \& Prach, 1993; Tockner et al., 2001; Zedler \& Kercher, 2004). Diese Einwanderung invasiver Arten wird vermehrt als eine ernstzunehmende Bedrohung für die jeweils einheimische Artenvielfalt wahrgenommen (Hejda et al., 2009; Hulme \& Bremner, 2006; Perglová et al., 2009), wobei die Auswirkungen der verschiedenen Arten sehr unterschiedlich bewertet werden (Hejda et al., 2009).

Impatiens glandulifera Royle gehört zu der Gruppe invasiver Neophyten, die auch in Auen artenarme Bestände bilden (Bartomeus et al., 2010; Beerling \& Perrins, 1993; Hulme \& Bremner, 2006; Perglová et al., 2009; Tickner et al., 2001; Zedler \& Kercher, 2004). Diese einjährige Art, die ihre natürliche Verbreitung in Auenbereichen des westlichen Himalayas hat, wird in vielen Ländern zu den bedenklichsten Neophyten gezählt und auch in der Literatur oftmals auf diese Weise dargestellt (Bartomeus et al., 2010; Beerling \& Perrins, 1993; Hulme \& Bremner, 2006; Perglová et al., 2009; Perrins et al., 1993). Dennoch wird in der aktuellen Forschung parallel dazu diskutiert, ob die Bedeutung von Impatiens glandulifera Royle nicht überschätzt wird. So wird beispielsweise argumentiert, dass das Indische Springkraut lediglich eine freie ökologische Nische innerhalb der Auenbestände besetzen würde oder maximal in Konkurrenz zu anderen dominanten Nitrophyten wie der Großen Brennnessel (Urtica dioica) treten würde (Ammer et al., 2011; Hejda \& Pyšek, 2006; Hejda et al., 2009; Kowarik, 2003).

Diese These wäre in dem Moment abzulehnen, wenn festgestellt werden würde, dass Impatiens glandulifera Royle aufgrund der arteigenen Merkmale einen anderen Konkurrenzdruck auf die einheimischen Arten der Weichholzauen ausübt und dadurch insbesondere die Verjüngung der typischen Baumarten, wie Salix alba, intensiver unterbinden würde. Da hierzu aktuell noch keine Studien publiziert wurden, besteht dringender Forschungsbedarf, um den Sachverhalt richtig einschätzen zu können. 


\subsubsection{Konkurrenzversuche unter kontrollierten Bedingungen und natürlichen Bedingungen}

Ein Konkurrenzversuch unter kontrollierten Bedingungen in Pflanztöpfen soll zunächst grundsätzlich darüber Aufschluss geben, inwieweit sich das Konkurrenzgefüge zwischen Salix alba und der einheimischen Ruderalart Urtica dioica im Vergleich zur Beziehung zwischen Salix alba und dem Neophyten Impatiens glandulifera Royle unterscheidet. Die Verwendung von Pflanztöpfen mit begrenztem Platzangebot für das entstehende Wurzelgefüge stellt trotz der Größe von Impatiens glandulifera Royle Individuen keine Problematik dar, weil das Wurzelwerk dieser Pflanzenart äußerst spärlich entwickelt ist (Burkhart \& Nentwig, 2008). Dadurch, dass die abiotischen Bedingungen standardisiert sind, können jegliche Unterschiede direkt auf die getesteten Parameter zurückgeführt werden. Daneben soll der Einfluss des hydrologischen Regimes auf das jeweilige Konkurrenzgefüge evaluiert werden. Zu diesem Zweck werden drei hydrologische Varianten getestet: keine Überflutung, eine Überflutung vor der Keimung von Salix alba, aber nach der Keimung von Urtica dioica und Impatiens glandulifera Royle und letztlich eine Überflutung nach der Keimung von Salix alba. Je hydrologisches Regime werden die Artenkombinationen Reinbestand aus Salix alba, Mischbestand aus Salix alba und Urtica dioica, Mischbestand aus Salix alba und Impatiens glandulifera Royle, Mischbestand aus Salix alba, Urtica dioica und Impatiens glandulifera Royle angelegt. Im Verlauf des Versuchs werden zur Bewertung des Konkurrenzdrucks auf Salix alba von Impatiens Glandulifera Royle und Urtica Dioica folgende Parameter erfasst: Anzahl erfolgreicher Keimungen von Salix alba sowie die Ausfallraten, Überlebensdauer und Vitalität von Salix alba Keimlingen und letztlich die oberirdische Biomasse von Salix alba Individuen. Um die Reaktion der Konkurrenten Impatiens glandulifera Royle und Urtica dioica auf die Variation des hydrologischen Regimes zu erfassen, wird auch ihre Vitalität erfasst.

Neben der Vermehrung durch Sämlinge stellt die vegetative Vermehrung von Weiden aus Bruchästen sowie Zweigfragmenten eine wichtige Vermehrungsstrategie für Weichholzauen dar. Die auf diese Weise entstehenden Jungbäume weisen bereits von Beginn an eine größere Höhe auf und zeigen auch nachfolgend ein schnelleres Wachstum als Sämlinge. Aus diesem Grund erreichen sie schneller die nötige Größe, um Überstauungen tolerieren zu können (Scholz et al., 2004). Diese Erkenntnis kann potenziell auf eine erfolgreiche Etablierung im Beisein von Impatiens glandulifera Royle übertragen werden. Um diese These zu überprüfen, wird der oben geschilderte Versuchsaufbau parallel mit Stecklingen statt Sämlingen durchgeführt.

Ergänzend zu den Versuchen unter kontrollierten Bedingungen wird das Konkurrenzverhältnis in Silberweidenbeständen unter natürlichen Verhältnissen 
betrachtet. Neben der Konkurrenzsituation liegt der Schwerpunkt hier zunächst auch auf der Frage, ob Weiden zur Keimung, wie häufig postuliert, frisch sedimentierte Rohbodenstandorte benötigen, oder ob auch lichtbegünstigte Bereiche in bestehenden Weichholzauenbeständen geeignet sind, wenn die oberirdische Biomasse von Konkurrenten entfernt wird. Wäre dies der Fall, scheint eine flächige Neuetablierung von Weiden realistischer, weil eine Überstauung der Fläche ohne morphologische Prozesse vor der Aussamung der Weiden ausreichend sein könnte, wenn die Dauer ausreicht, die etablierte Vegetation ausreichend zu schwächen. Zur Klärung der Frage werden ein Quadratmeter große Testflächen an wenig beschatteten Standorten in Weichholzauenbeständen eingerichtet. Es werden drei Flächentypen gewählt: Flächen mit der Dominanz von Impatiens glandulifera Royle, Flächen mit Dominanz von Urtica dioica und Flächen ohne extreme Dominanz. Auf allen Flächen werden gezielt Weidensamen eingebracht. Auf der Hälfte der Aufnahmeflächen wird zudem die gesamte oberirdische Biomasse anderer Pflanzen entfernt, die andere Hälfte bleibt unbehandelt. Im Verlauf des Versuchs werden entsprechend dem Versuch unter kontrollierten Bedingungen folgende Parameter erfasst: Anzahl erfolgreicher Keimungen von Salix alba, Ausfallraten, Vitalität und Überlebensdauer von Keimlingen sowie die oberirdische Biomasse der Salix alba Individuen.

\subsubsection{Versuche bezüglich des Einflusses der allelopathischen Wirkung von Impatiens glandulifera Royle auf den Keimungserfolg von Weiden}

Im Versuch von Dericks und Lösch (unveröffentlicht, nach Frey \& Lösch, 2004) (wiederholt im Zuge des BIOLOG-Projekts, BIOLOG, 2003), wurde die allelopathische Wirkung eines Rohpresssafts aus Impatiens glandulifera Royle Pflanzen auf Kresse untersucht und im Zuge dessen bestätigt. Im BIOLOG-Projekt führte eine 1:1 Verdünnung des Rohpresssaftes zu einer Keimungsrate von 34\% und unverdünnter Extrakt zu einem völligen Ausbleiben von Keimungen der Kressesamen, während die Rate der Kressekeimung auf destilliertem Wasser gesättigtem Filterpapier binnen 1 Woche nahezu $100 \%$ erreicht hatte (BIOLOG, 2003). Angelehnt an diesen Versuchsaufbau soll eine potenzielle allelopathische Wirkung von Impatiens glanulidera Royle auf den Keimungserfolg von Sämlingen und Anwuchserfolg von Stecklingen von Salix alba getestet werden. Hierzu werden Individuen von Impatiens glandulifera Royle geerntet und ein Rohpresssaft daraus hergestellt, der vergleichbare Auswirkungen wie verrottende Impatiens glandulifera Royle Pflanzen in der Natur auf den Boden hat. Mit diesem Extrakt werden Stecklinge, Samen und eventuelle spätere Keimlinge von Salix alba unter kontrollierten Bedingungen in Keimschalen gegossen. Um die Auswirkungen auf den Etablierungserfolg von Weiden zu evalu- 
ieren, werden die erfolgreichen Keimungen, die Überlebensdauer der Individuen und die Vitalität im Vergleich zu einer Kontrollgruppe von ausgebrachten Salix alba Samen und Stecklingen, die diesem Extrakt nicht ausgesetzt werden, erfasst.

\section{$4 \quad$ Perspektive für Weichholzauenbestände in gestauten Flussabschnitten}

Durch intensive Forschung im semiariden Nordwesten Amerikas (Mahoney \& Rood, 1998; Rood, 2000; Rood et al., 2003; Stella et al., 2010) konnte dort durch die künstliche Bereitstellung passender Abflussverhältnisse eine deutliche Verbesserung der Fluss- und Auenökosysteme in stark anthropogen überprägten Flussabschnitten mit Staubereichen erreicht werden. Pappelverjüngung kann dort mittlerweile durch dieses ökologische Management der Staudämme gezielt hervorgerufen werden. Diese Ergebnisse stimmen durchaus optimistisch für die Annahme, dass die Entwicklung einer "Recruitment box“ auch in humiden Klimaten eine positive Trendwende anstoßen kann. Im semiariden Raum konnte das Entwicklungspotenzial allerdings durch Erhöhung des Restwasserabflusses und verlangsamten Abflussrückgang unterhalb der Staudämme ausgeschöpft werden. Der limitierende Faktor, die Wasserverfügbarkeit, ist für diesen Ansatz nicht von tiefgreifender Bedeutung, weil die Steuermöglichkeiten ohnehin auf ausreichend feuchte Jahre beschränkt sind und die Pappelverjüngung auch in natürlichen Systemen nicht jährlich, sondern lediglich episodisch (Mahoney \& Rood, 1998; Rood et al., 2003) in 5-10 Jahresintervallen (Mahoney \& Rood, 1993) beziehungsweise in einem Intervall von 3-10 Jahren mit mittleren bis hohen Frühjahrshochwässern (Cordes et al., 1997; Mahoney \& Rood, 1998; Scott et al., 1996) gelingt.

Im humiden Raum dagegen ist die Wasserverfügbarkeit insgesamt nicht limitierend, sobald das Staumanagement nicht mehr ausschließlich auf optimierte Stromproduktion ausgerichtet wird, sondern zeitweise Einbußen bei der Energiegewinnung zu Gunsten des Naturschutzes gemacht würden. Viel schwerwiegender zeigt sich der Mangel an Raum für den Fluss und seine Entfaltung. Dieser Zustand hat sich trotz wiederkehrender Beteuerungen seitens der Politik nach Hochwasserereignissen kaum verbessert. Wenn durch ökologisch bewusst erhöhten Flusswasserstand in zu nah herangerückter Bebauung steigende Grundwasserständeauftreten, wird dies nicht akzeptiert werden, so dass sich jegliche Auswirkungen auf den Raum innerhalb der Deichgrenzen beschränken müssen. Im Unterwasserbereich von Staustufen sind Fluss und Aue durch intensive Tiefenerosion meistens bereits so deutlich voneinander getrennt, dass eine ausreichend lange Überstauung durch 
den Hauptfluss zur Schädigung der Konkurrenzvegetation nicht möglich ist. Hier blieben zwei Möglichkeiten: ein kleines ökologisches Fließgewässer durch die Auenbereiche künstlich schaffen und dieses dann entsprechend der Recruitment Box gezielt steuern und auch aufstauen. Da der Hauptfluss aber stets über das Grundwasser als Entwässerungsgraben fungieren wird, werden dieser Variante nur geringe Erfolgschancen prognostiziert.

Die zweite Möglichkeit ist, das Gelände entweder künstlich oder durch Erosionsarbeit des kleinen ökologischen Fließgewässers entsprechend auf Flussniveau abzutragen. Im unmittelbaren Staubereich flussaufwärts der Stauhaltungen ist der Fluss meist komplett eingedeicht und mit seiner Aue nur über einen kleinen Entwässerungsgraben in Verbindung. Der Grundwasserspiegel im Umland ist dabei generell ausreichend hoch anstehend, aber bisher entsprechend der Abflusssteuerung zu statisch für eine Auenentwicklung. Ökologische Flutungen scheinen in diesem Bereich bis zum Ende der Stauwurzel aufgrund der Nähe des Flusswasserspiegels zum Auenniveau umsetzbar.

Aufgrund der geschilderten Problematik sind viele Renaturierungsprojekte nach wie vor oft noch auf kleine Teilbereiche und künstliche, sehr kostenintensive (Bau-) Maßnahmen, wie Polder oder Pflanzungen beschränkt, die nachfolgend permanente Pflege bedürfen und oft fehlschlagen (Alpert et al., 1999; Malanson, 1993). Gerade Naturschutzmaßnahmen, die auf statische Erhaltung bestimmter Biotope oder Arten an definierter Stelle abzielen, stehen der großen Dynamik intakter Auen gegenüber und sind nicht Erfolg versprechend (Korn et al., 2005).

Das Beispiel des semiariden Nordwesten Amerikas zeigt aber deutlich, dass nicht allein das Vorhandensein von Dämmen die Auenlebensräume entscheidend schädigt, sondern ihr Abflussmanagement ausschlaggebend ist und zumindest im Bezug auf die Vegetation der Weichholzauen Potenzial zur Rehabilitierung besteht.

Wie zu Beginn bereits ausführlich geschildert, existieren in Deutschland und europaweit nur noch vereinzelte kleinste Restflächen intakter Auen. Daher gilt es hier zunächst dem Erhalt dieser noch funktions- und leistungsfähigen (Fluss-) Ökosysteme Priorität bei der Nutzung der mitteleuropäischen Landschaften einzuräumen, denn die Schwächung der Säulen Naturschutz und Biodiversität ist in diesen Fällen stärker zu bewerten als der positive Einfluss auf Klimaschutz und Ressourcenschutz. Dies gilt ungeachtet dessen, ob durch entsprechende Gestaltungsmöglichkeiten und Steuerungen der negative Einfluss minimiert werden kann, denn ,ein ,restored environment' (besitzt) [zwar] mehr Wert (...), als ein , degraded environment', aber (stets) weniger Wert als ein weitgehend unbeeinflusste[s], original environment"“ (Zerbe \& Wiegleb, 2009: 431). 


\section{Literatur}

Alpert, P., Griggs, F. T. \& Peterson, D. R. (1999). Riparian forest restoration along large rivers: Initial results from the Sacramento River Project. Restoration Ecology: 360-368.

Alldredge, B. \& Moore, G. (2012). Assessment of riparian vegetation sensitivity to river hydrology downstream a major texas dam. River Research and application, River Res. Applic (2012).

Ammer, Ch., Schall, P., Wördehoff, R., Lamatsch, K. \& Bachmann, M. (2011). Does tree seedling growth and survival require weeding of Himalayan balsam (Impatiens glandulifera)? European Journal of Forest Research, 130(1): 107-116.

Augustin, J. (2001). Emission, Aufnahme und Klimarelevanz von Spurengasen. In: M. Succow \& H. Joosten (Hrsg.), Landschaftsökologische Moorkunde. Stuttgart: Schweizerbart.

Bartomeus, I., Vilà, M. \& Steffan-Dewenter, I. (2010). Combined effects of Impatiens glandulifera invasion and landscape structure on native plant pollination. Journal of Ecology 2010, 98(2): 440-450.

Bayerisches Landesamt für Umwelt (LFU) \& Bayerische Landesanstalt für Wald und Forstwirtshaft (LWF) (2004). HANDBUCH der Lebensraumtypen nach Anhang I der Fauna-Flora-Habitat-Richtlinie in Bayern. 165 S. + Anhang, Augsburg \& Freising-Weihenstephan.

Bayerische Staatsregierung (2011). Bayerisches Energiekonzept „Energie innovativ“. München.

Beerling, D.J. \& Perrins, J.M. (1993). Biological flora of British Isles: Impatiens glandulifera. Journal of Ecology, 81: 367-382.

Biolog (2003). Zwischenbericht 2003 zum BIOLOG-Projekt.

Bragg, D. C. (2000). Simulating catastrophic and individualistic large woody debris recruitment for a small riparian system. Ecology, 81(5): 1383-1394.

Braun-Blanquet, J. (1964). Pflanzensoziologie. Wien/New York: Springer.

Brunotte, E., Dister, E., Günther-Diringer, D., Koenzen, U. \& Mehl, D. (2009). Flussauen in Deutschland. Erfassung und Bewertung des Auenzustandes. Bonn: BfN Schriftenvertrieb.

Bundesanstalt für Gewässerkunde (2012). Vegetation der Donauaue zwischen Straubing und Vilshofen. Standortpotenzial für die Auenvegetation des Ist-Zustands und der Ausbauvarianten. Unveröffentlicht.

Bundesministerium Wirtschaft und Technologie (2012). Energiewende in Deutschland. Berlin. Bundesumweltministerium (2007). Nationale Strategie zur biologischen Vielfalt. Berlin.

Bunn, S. E. \& Arthington, A. H. (2002). Basic principles and ecological consequences of altered flow regimes for aquatic biodiversity. Environmental Management, 30: 492-507.

Burkhart, K. \& Nentwig, W. (2008). Control of Impatiens glandulifera (Balsaminaceae) by Antagonists in its invaded range? Invasive Plant Science and Management, 1(4): 352-358.

Chasek, P., David, L. D. \& Welsh Brown, J. (2006). Handbuch Globale Umweltpolitik. Berlin: Parthas.

Cordes, L. D., Hughes, F. M. R. \& Getty, M. (1997). Factors affecting the regeneration and distribution of riparian woodlands along a northern prairie river: The Red Deer River, Alberta, Canada. Journal of Biogeography, 24: 675-695.

Cyffka, B. \& Haas, F. (2008). Erosion without sediment supply? The crux of a floodplain restoration project downstream dammed-up headwaters. In: J. Schmidt (Hrsg.), Sediment Dynamics in Changing Environments (pp. 477-484). Wallingford: IAHS Press. 
Damm, Ch. et al. (2011). Auenschutz - Hochwasserschutz - Wasserkraftnutzung. Beispiele für eine ökologisch vorbildliche Praxis. Bonn/Bad Godesberg: BfN Schriftenvertrieb.

Debano, L. F. \& Schmidt, L. J. (1990). Potential for enhancing riparian habitats in the southwestern Unites States with watershed practices. Forest Ecology and Management, 33/34: 385-403.

Décamps, H., Planty-Tabacchi, A.M. \& Tabacchi, E. (1995). Changes in the hydrological regime and invasions by plant species along riparian systems of the Adour River, France. Regulated Rivers: Research and Management, 11: 23-33.

Detering, U. (2000). Das Gewässerauenprogramm NRW am Beispiel der oberen Lippe. Bundesamt für Naturschutz (Hrsg.): Renaturierung von Bächen, Flüssen und Strömen. Angewandte Landschaftsökologie, 37: 153-162.

Deutscher Bundestag (1994). Die Industriegesellschaft gestalten. Perspektiven für einen nachhaltigen Umgang mit Stoff- und Materialströmen. Bericht der Enquete-Kommission „Schutz des Menschen und der Umwelt - Bewertungskriterien und Perspektiven für Umweltverträgliche Stoffkreisläufe in der Industriegesellschaft“ des 12. Deutschen Bundestages. Bonn: Economica Verlag.

Diefenbacher, H., Karcher, H., Stahmer, C. \& Teichert, V. (1997). Nachhaltige Wirtschaftsentwicklung im regionalen Bereich - ein System von ökologischen, ökonomischen und sozialen Indikatoren. Heidelberg: Forschungsstätte der Evangelischen Studiengemeinschaft.

Dister, E. (1983). Zur Hochwassertoleranz von Auenwaldbäumen an lehmigen Standorten. Verhandlungen der Gesellschaft für Ökologie, 10: 325-336.

Dister, E. (1991). Situation der Flußauen in der Bundesrepublik Deutschland. Laufener Seminarbeiträge, 4: 8-16.

Dister, E. (1998). Die Bedeutung natürlicher Flussdynamik am Beispiel von Loire und Allier. Schriftenreihe Landschaftspflege und Naturschutz, 56: 67-78.

Dörfer, K. (2000). Artenschutz durch Stabilisierung des Lebensraums - ein Irrweg? Angewandte Landschaftsökologie, 37: 49-58.

Ellenberg, H. \& Leuschner, Ch. (2010). Vegetation Mitteleuropas mit den Alpen: In ökologischer, dynamischer und historischer Sicht. Stuttgart: UTB.

Foeckler, F. \& Bohle, W. (1991). Fließgewässer und ihre Auen - prädestinierte Standorte ökologischer und naturschutzfachlicher Grundlagenforschung. In: K. Henle \& G. Kaule (Hrsg.), Arten- und Biotopschutzforschung in Deutschland. - Berichte aus der ökologischen Forschung, Bd. 4 (pp. 236-266). Jülich: KFA.

Forstwirtschaft (LWF) (2004). HANDBUCH der Lebensraumtypen nach Anhang I der Fauna-Flora-Habitat-Richtlinie in Bayern (mit Anhang). Augsburg/Freising-Weihenstephan.

Frei, Ch., Schöll, R., Fukutome, S., Schmidli, J. \& Vidale, P. L. (2006). Future change of precipitation extremes in Europe: Intercomparison of scenarios from regional climate models. Journal of geophysical research 111. D06105. ISSN 0148-0227 doi: 10.1029/2005JD005965.

Frey, W. \& Lösch, R. (2004). Lehrbuch der Geobotanik: Pflanze und Vegetation in Raum und Zeit. München: Spektrum Akademischer Verlag.

Gerken, B. (1988). Auen - verborgene Lebensadern der Natur. Freiburg: Rombach.

Gerken, B., Böttcher, H., Leifeld, D., Lohr, M., Dörfer, K. \& Leushacke-Schneider, Ch. (2000). Beurteilung von Regenerationsmaßnahmen durch vegetationskundliche und faunistische Untersuchungen - Beispiele aus der Oberweserniederung. Angewandte Landschaftsökologie, 37: 205-216.

Hejda, M. \& Pyšek, P. (2006). What is the impact of Impatiens glandulifera on species diversity of invaded riparian vegetation? Biological Conservation, 132: 143-152. 
Hejda, M., Pyšek, P. \& Jarošík, V. (2009). Impact of invasive plants on the species richness, diversity and composition of invaded communities. Journal of Ecology 97: 393-403.

Henrichfreise, A. (2000). Zur Erfassung von Grundwasserstandsschwankungen in Flußauen als Grundlage für Landeskultur und Planung - Beispiele von der Donau. Angewandte Landschaftsökologie, 37: 205-216.

Hood, W. G. \& Naiman, R. J. (2000). Vulnerability of riparian zones to invasion by exotic woody plants. Plant Ecology, 148: 105-114.

Hughes, F. M. R., Adams, W. M., Muller, E., Nilsson, C., Richards, K. S. \& Barsoum, N. (2001). The importance of different scale processes for the restoration of floodplain woodlands. Regulated Rivers - Research and Management, 17: 325-345.

Hulme, P.E. \& Bremner, E.T. (2006). Assessing the impact of Impatiens glandu- lifera on riparian habitats: partitioning diversity components following species removal. Journal of Applied Ecology, 43: 43-50.

IPCC (2007). Climate Change 2007: the physical basis. Summary of policymakers. Contributions of working group I to the fourth assessment report oft he Intergovernmentl Panel of Climate Change. https://ipcc.ch/SPM-2feb07.pdf. Zugegriffen: 10.01.2016.

Jürging, P. \& Patt, H. (Hrsg.). (2005). Fließgewässer- und Auenentwicklung Grundlagen und Erfahrungen. Berlin/Heidelberg: Springer.

Kasperek, G. (2004). Fluctuations in numbers of neophytes, especially Impatiens glandulifera, in permanent plots in a west German floodplain during 13 years. In: I. Kühn \& S. Klotz (Hrsg.), Biological Invasions: Challenges for Science (pp. 27-37). NEOBIOTA 3: 27-37.

Korn, N., Jessel, B., Hasch, B. \& Mühlinghaus, R. (2005). Flussauen und Wasserrahmenrichtlinie. Bedeutung der Flussauen für die Umsetzung der europäischen Wasserrahmenrichtlinie - Handlungsempfehlungen für Naturchutz und Wasserwirtschaft. Bonn/ Bad Godesberg: BfN Schriftenvertrieb.

Kowarik, I. (2003). Biologische Invasionen: Neophyten und Neozoen in Mitteleuropa. Stuttgart: Ulmer Eugen Verlag.

Kramer, K., Vreugdenhill, S. J. \& van der Werf, D. C. (2008). Effects of flooding on the recruitment, damage and mortality of riparian tree species: A field and simulation study on the Rhine floodplain. Forest Ecology and Management, 255: 3893-3903.

Kunstmann, H., Schneider, K., Forkel, R. \& Knoche, R. (2004). Impact analysis of climate change for an Alpine catchment using high resolution dynamic downscaling of ECHAM4 time slices. Hydrology and Earth System Sciences, 8: 1030-1044.

Lambdon, P.W. et al. (2008). Alien flora of Europe: species diversity, temporal trends, geographical patterns and research needs. Preslia 80: 101-149.

Londo, G. (1975). De decimale scaale foor vegetatiekundelige opnamen van permanente Kwadraten. Gorteria, 7: 101-106.

Mahoney, J. M. \& Rood, S. B. (1998). Streamflow requirements for cottonwood seedling recruitment: An integrative model. Wetlands, 18: 634-645.

Mahoney, J.M. \& Rood, S.B. (1993). A model for assessing the effects of altered river flows on the recruitment of riparian cottonwoods. In: B. Tellman, H.J. Cortner, M.G. Wallace, L.E. DeBano \& R.H. Hamre (Hrsg.), Riparian Management: Common Threads and Shared Interests (pp. 228-232). Albuquerque, NM: USDA Forest Service.

Malanson, J. P. (1993). Riparian Landscapes. Cambridge, UK: Cambridge University Press. Margraf, Ch. (2004). Die Vegetationsentwicklung der Donauauen zwischen Ingolstadt und Neuburg. Hoppea, 65: 295-703. 
Mosner, E., Schneider, S., Conradt, T., Lehmann, B., Hatterman, F. F. \& Leyer, I. (2008). Floodplain forests and climate change - current state and future pattern. 6th European Conference on Ecological Restoration. Ghent, Belgium, 8-12/09/2008. 1-4.

Mößmer, E.-M. (2000). Wald - Wasser - Leben. Köln: Stiftung Wald in Not.

Müller, D., Schöl, A., Bergfeld, T. \& Strunk, Y. (Hrsg.). (2006). Staugeregelte Flüsse in Deutschland. Wasserwirtschaftliche und ökologische Zusammenhänge. Limnologie aktuell (Bd. 12). Stuttgart: Schweizerbart'sche Verlagsbuchhandlung.

Oberdorfer, E. (Hrsg.) (1992). Süddeutsche Pflanzengesellschaften. Teil IV: Wälder und Gebüsche. Textband. Jena: Fischer.

Pasquale, N., Perona, P., Francis, R. \& Burlando, P. (2012). Effects of streamflow variability on the vertical root density distribution of willow cutting experiments. Ecological Engineering, 40: 167-172.

Peper, J., Horchler, P. \& Schleuter, M. (2012): Vegetation der Donauaue zwischen Straubing und Vilshofen. Standortpotenzial für die Auenvegetation des Ist-Zustands und der Ausbauvarianten. Koblenz.

Perglová, I., Pergl, J., Skálová, H., Moravcová, L., Jarošík, V. \& Pyšek, P. (2009). Differences in germination and seedling establishment of alien and native Impatiens species. Preslia, 81: $357-375$.

Perrins, J., Fitter, A. \& Williamson, M. (1993). Population biology and rates of invasion of three introduced Impatiens species in the British Isles. Journal of Biogeography, 20: 3-44.

Pfadenhauer, J. (1997). Vegetationsökologie - ein Skriptum (2. Aufl.). Eching: IHW-Verlag.

Pyšek, P. \& Prach, K. (1993). Plant invasions and the role of riparian habitats: a comparison of four species alien to central Europe. Journal of Biogeography, 20: 413-420.

Richardson, D. M. et al. (2007). Riparian vegetation: degradation, alien plant invasions, and restoration prospects. Diversity and Distributions, 13: 126-139.

Riecken, U., Finck, P., Raths, U., Schröder, E. \& Ssymank, A. (2006). Rote Liste der gefährdeten Biotoptypen Deutschlands. Naturschutz und biologische Vielfalt, 34: 318.

Robinson, A. (2000). Flood Protection through Floodplain Channel Restoration - Examples from the Upper Weser Region. Angewandte Landschaftsökologie, 37: 101-111.

Rood, S. B. (2000). Revised instream flow regulation enables cottonwood recruitment along the St. Mary River, Alberta, Canada. Rivers, 7(2): 109-125.

Rood, S. B. et al. (2003). Flows for Floodplain Forests: A Successful Riparian Restoration. BioScience, 53(7): 647-656.

Rowell, D. P. \& Jones, R. G. (2006). Causes and uncertainty of future summer drying over Europe. Climate Dynamics, 27: 281-299.

Sautter, R. (2003). Waldgesellschaften in Bayern. Vegetationskundliche und forstgeschichtliche Darstellung der natürlichen und naturnahen Waldgesellschaften. Landsberg: ecomed.

Scholten, M. et al. (2005). The Elbe River in Germany - present state, conflicts, ad perspectives of rehabilitation. Archiv für Hydrobiologie, Large Rivers 15: 579-602.

Scholz, M., Henle, K., Dzick, F., Stab, S., Foeckler, F. (Hrsg.) (2009). Entwicklung von Indikationssystemen am Beispiel der Elbaue. Stuttgart: Verlag Eugen Ulmer.

Scholz, M., Stab, S., Dziock, F. \& Henle, K. (Hrsg.) (2004). Lebensräume der Elbe und ihrer Auen. Berlin: Weißensee-Verlag,

Schubert, R., Hilbigm, W. \& Klotz, S. (2001). Bestimmungsbuch der Pflanzengesellschaften Deutschlands. Heidelberg: Springer. 
Schwartz, R. (2001). Die Böden der Elbaue bei Lenzen und ihre möglichen Veränderungen nach Rückdeichung. Dissertation am Fachbereich Geowissenschaften der Universität Hamburg, Hamburger Bodenkundliche Arbeiten, 48.

Scott, M. L., Auble, G. T. \& Friedman, J. M. (1996). Fluvial process and the establishment of bottomland trees. Geomorphology, 14: 327-339.

Seibert, P. (1971). Pflanzensoziologisches Gutachten über die Donauauen des Wittelsbacher Ausgleichsfonds. Unveröffentlichtes Gutachten. München.

Spindler, E. (o. J.). Geschichte der Nachhaltigkeit. Vom Werden und Wirken eines beliebten Begriffs. www.nachhaltigkeit.info/media/1326279587phpeJPyvC.pdf. Zugegriffen: 14.01.2016.

Stahlmann, V. (2008). Lernziel: Ökonomie der Nachhaltigkeit. Eine anwendungsorientierte Übersicht. München: Oekom Verlag.

Stella, J. C. (2005). A mechanistic model to evaluate and improve riparian restoration success. CALFED Science Fellowshop Post-Award report, Project R/SF-2. https:/caseagrant. ucsd.edu/publication/a-mechanistic-model-to-evaluate-and-improve-riparian-restoration-success. Zugegriffen: 10.01.2016.

Stella, J. C., Battles, J. J., McBride, J. R. \& Bruce, K. O. (2010). Riparian Seedling Mortality from Simulated Water Table Recession, and the Design of Sustainable Flow Regimes on Regulated Rivers. Restoration Ecology, 18(S2),284-294.

Stillwater Sciences (2006). Restoring recruitment processes for riparian cottonwoods and willows: a field-calibrated predictive model for the lower San Joaquin Basin. Pre pared for CALFED Bay-Delta Ecosystem Restoration Program, Sacramento, California. Prepared by Stillwater Sciences and Dr. John Stella, in conjunction with Dr. John Battles and Dr. Joe McBride, Department of Environmental Science, Policy, and Management, University of California, Berkeley.

Tickner, D. P., Angold, P. G., Gurnell, A. M., Mountford, J. O. \& Sparks, T. (2001). Hydrology as an influence on invasion: experimental investigations into competition between the alien Impatiens glandulifera and the native Urtica dioica in the UK. Plant invasions: species ecology and ecosystem management, 159-167.

Tockner, K., Malard, F. \& Ward, J. V. (2000). An extension of the flood pulse concept. Hydrological processes, 14: 2861-2883.

Volk, H. (2000). Neue Ergebnisse der Auewaldforschung am Rhein. Angewandte Landschaftsökologie, 37: 23-32.

Walentowski, H., Ewald, J., Fischer, A., Kölling, Ch. \& Türk, W. (2004). Handbuch der natürlichen Waldgesellschaften Bayern. Freising: Geobotanica.

Wenger, E. L., Zinke, A. \& Gutzweiler, K.-A. (1990). Present situation of European floodplain forests. Forest Ecology and Management, 33/34: 5-12.

Zahlheimer, W. A. (1979). Vegetationsstudien in den Donauauen zwischen Regensburg und Straubing als Grundlage für den Naturschutz. Hoppea, 38: 3-398.

Zedler, J. B. \& Kercher, S. (2004). Causes and Consequences of Invasive Plants in Wetlands: Opportunities, Opportunists, and Outcomes. Critical Review in Plant Sciences, 23(5): 431-452.

Zerbe, S. \& Wiegleb, G. (Hrsg.). (2009). Renaturierung von Ökosystemen in Mitteleuropa. Heidelberg: Springer. 
Open Access Dieses Kapitel wird unter der Creative Commons Namensnennung - Nicht kommerziell 2.5 International Lizenz (http://creativecommons.org/licenses/by-nc/2.5/deed. de) veröffentlicht, welche für nicht kommerzielle Zwecke die Nutzung, Verbreitung und Wiedergabe in jeglichem Medium und Format erlaubt, sofern Sie den/die ursprünglichen Autor(en), den Titel des Werks und die Quelle ordnungsgemäß nennen, einen Link zur Creative Commons Lizenz beifügen und im Falle einer Abwandlung durch einen entsprechenden Hinweis deutlich erkennbar machen, dass Änderungen vorgenommen wurden.

Die in diesem Kapitel enthaltenen Bilder und sonstiges Drittmaterial unterliegen ebenfalls der genannten Creative Commons Lizenz, sofern sich aus der Abbildungslegende nichts anderes ergibt. Sofern das betreffende Material nicht unter der genannten Creative Commons Lizenz steht und die betreffende Handlung nicht nach gesetzlichen Vorschriften erlaubt ist, ist auch für die oben aufgeführten nicht-kommerziellen Weiterverwendungen des Materials die Einwilligung des jeweiligen Rechteinhabers einzuholen. 